\title{
Autofocusing Algorithm Selection in Computer Microscopy
}

\author{
Yu Sun \\ Department of Mechanical and Industrial Engineering \\ Institute of Biomaterials and Biomedical Engineering \\ University of Toronto \\ Toronto, M5S 3G8, Canada \\ sun@mie.utoronto.ca
}

\begin{abstract}
Autofocusing is a fundamental technology for automated biological and biomedical analyses and is indispensable for routine use of microscopes on a large scale. This paper presents a comprehensive comparison study of 18 focus algorithms in which a total of 139,000 microscope images are analyzed. Six samples were used with three observation methods (bright field, phase contrast, and differential interference contrast (DIC)) under two magnifications (100X and 400X). A ranking methodology is proposed, based on which the 18 focus algorithms are ranked. Image pre-processing is also conducted to extensively reveal the performance and robustness of the focus algorithms. The presented guidelines allow for the selection of the optimal focus algorithm for different microscopy applications.
\end{abstract}

Index Terms - autofocusing, microscopy, ranking, selection.

\section{INTRODUCTION}

Autofocusing is a fundamental technology for automated biological and biomedical applications, such as in high throughput screening for the pharmaceutical industry and in autonomous microrobotic cell manipulation [1]. Reliable autofocusing methods are also indispensable for routine use of microscopes on a large scale as in the microassembly of hybrid microelectromechanical systems (MEMS) [2].

Although autofocusing is a long standing topic in the literature and a variety of focus algorithms have been proposed, the selection of an appropriate focus algorithm for specific experimental microscopy conditions remains ad hoc and time consuming. Based on the comparison results of 13 focus algorithms operating on fluorescence images, it was found by [3] that AutoCorrelation [4][5] is the optimal focus algorithm for fluorescence microscopy applications. However, AutoCorrelation was not found to be the optimal focus algorithm in this study for microscopy images under bright field, phase contrast, or differential interference contrast (DIC), thus motivating this study.

This paper presents a systematic evaluation of 18 widely used focus algorithms and comprehensive comparison results by processing a total of 139,000 microscope images. Six samples were used in experiments with three observation methods (bright field, phase contrast, and DIC) and two magnifications (100X and 400X). Besides the criteria of monotonicity and single modality for evaluating focus algorithms, three other criteria are adopted to systematically evaluate the performance of the 18 focus algorithms. In order to extensively study the algorithms' performance, filtering (pre-processing) operations including sub-sampling, low-pass filtering, and random noise addition are conducted on images
Stefan Duthaler and Bradley J. Nelson

Institute of Robotics and Intelligent Systems

Swiss Federal Institute of Technology (ETH-Zurich)

Zurich,8092, Switzerland

bnelson@ethz.ch

before the focus algorithms are applied. Based on the large amount of experimental data, the performance of the focus algorithms is analyzed and compared using individual criteria and an overall ranking is determined. Subsequently, guidelines for selecting the optimal focus algorithm are obtained.

(a)

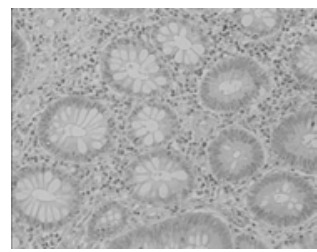

(b)

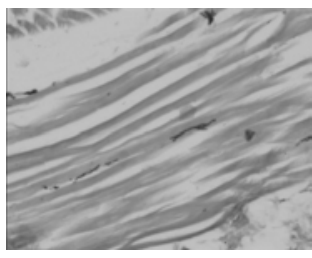

(c)
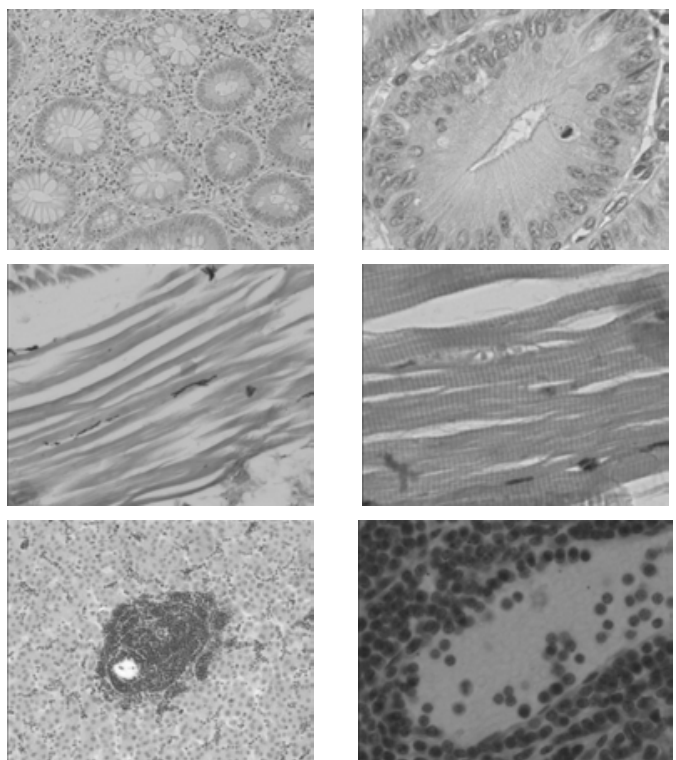

(d)
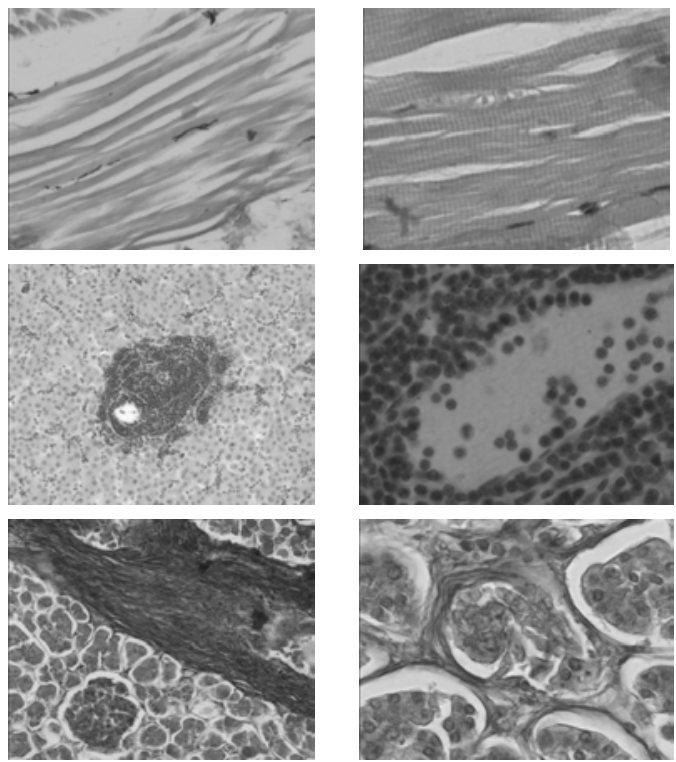

(e)
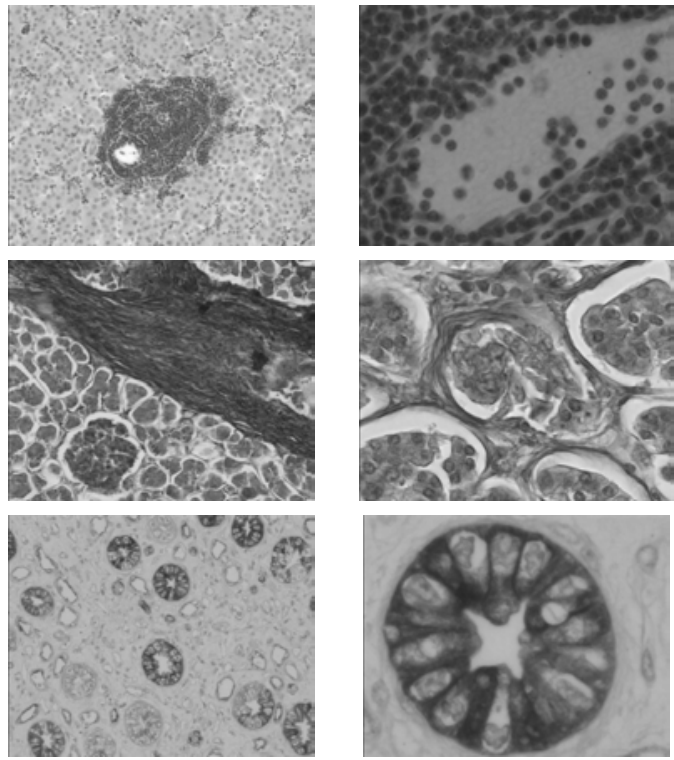

(f)
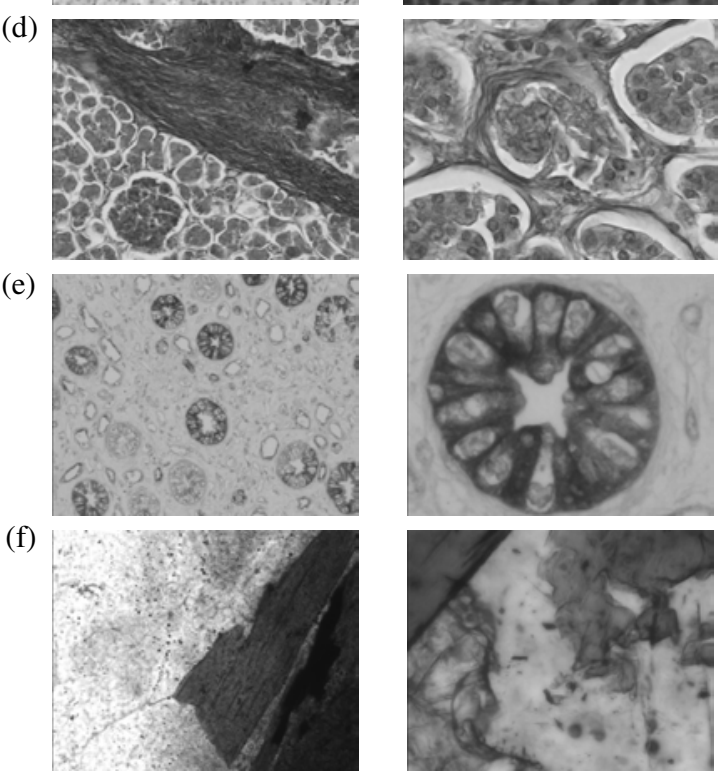

Fig. 1 Bright field images of the samples with 100X (left column) and 400X (right column). From top to bottom: Sample 1: Adenomatosis of the colon. Sample 2: Muscles of a baby snake (asp). Sample 3: Liver cells. Sample 4: Pancreas cells. Sample 5: Kidney cells. Sample 6: Ground stone. 
(a)

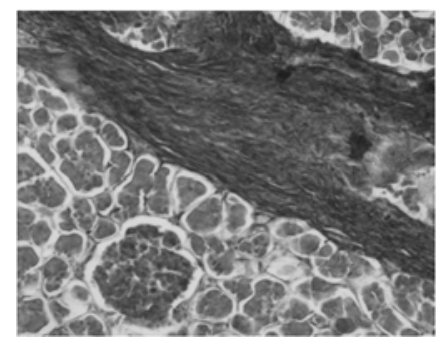

(b)

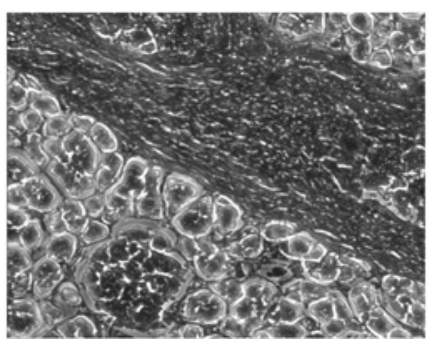

(c)

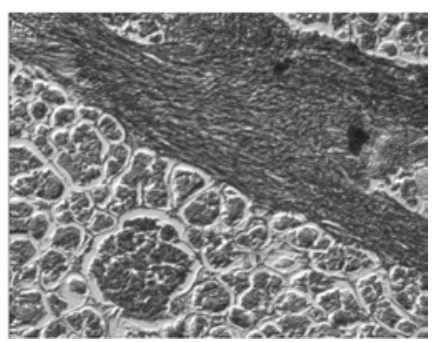

Fig. 2 Images of pancreas cells (Sample 4 in Fig. 1) under: (a) bright field; (b) phase contrast; (c) differential interference contrast (DIC).

\section{MATERIALS AND METHODS}

The six samples shown in Fig. 1 were used in the experiments for testing the 18 focus algorithms. The thickness of the samples is $2-6 \mu \mathrm{m}$. These samples were chosen because they embody a variety of information, from which more general conclusions for selecting the optimal focus algorithm can be reached. The larger the information content, the easier the focusing.

The experimental setup consists of a motorized inverted microscope (Olympus IX81), a framegrabber (Matrox MeteorII), and a CCD camera (Sony XC-HR50). The motorized inverted microscope has a minimum focus step of $0.01 \mu \mathrm{m}$. Two objectives were used, a 10X objective (Olympus UPlanFl, $3 \mu \mathrm{m}$ depth of field, NA 0.3 ) and a $40 \mathrm{X}$ objective (Olympus LCPlanFl, $1 \mu \mathrm{m}$ depth of field, NA 0.6). The microscope provides three observation methods, including bright field, phase contrast, and DIC. Fig. 2 shows pancreas cells (sample 4) under these three optical conditions.

The 100X image sets were obtained in $1 \mu \mathrm{m}$ steps, amounting to 400 images per sample. The $400 \mathrm{X}$ image sets were obtained in $0.25 \mu \mathrm{m}$ steps within a smaller range, amounting to 550 images per sample. Applying the three observation methods and two magnifications to the six samples, 36 image sets were obtained with each set having either 400 images or 550 images. A total of 139,500 images $(640 \times 494)$ were processed and analyzed on a PC $(2.53 \mathrm{GHz}$ CPU and 512MB RAM). Computation time, discussed in the Results and Discussion section, does not exceed 30ms for almost all the focus algorithms tested.

\section{FOCUS ALGORITHMS}

Many focus algorithms have been proposed and compared in the literature. The output of an ideal focus algorithm is defined as having a maximum value at the best focused image/position and decreasing as defocus increases. The 18 widely used focus algorithms in the literature that were tested and compared in this study can be classified into four groups.

\section{A. Derivative-Based Algorithms}

These algorithms assume that well-focused images have more high-frequency content than defocused images. Neighboring pixels in images with high-frequency content have large differences in intensity. The larger these intensity changes, the sharper the edges. In order to apply high pass filtering, these algorithms apply convolution masks to an image to obtain derivatives. Different norms are subsequently used to compute the magnitude of the derivative vectors. These algorithms are sensitive to high-frequency noise.

1) Threshold absolute gradient [3]: It sums the absolute value of the first derivative that is larger than a threshold $\theta$.

$$
F_{\text {th_-grad }}=\sum_{\text {Height Width }}|i(x+1, y)-i(x, y)|
$$

where $|i(x+1, y)-i(x, y)| \geq \theta$, and $i(x, y)$ is the grey level intensity of pixel $(x, y)$.

2) Squared gradient [3]: This algorithm sums squared differences, making larger gradients exert more influence.

$$
F_{s q_{-} \text {grad }}=\sum_{\text {Height Width }} \sum_{i}(i(x+1, y)-i(x, y))^{2}
$$

where $(i(x+1, y)-i(x, y))^{2} \geq \theta$.

3) Brenner gradient [6]: This algorithm computes the first difference between a pixel and its neighbor with a horizontal/vertical distance of 2 .

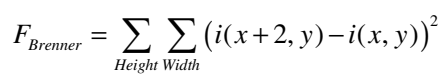

where $(i(x+2, y)-i(x, y))^{2} \geq \theta$.

4) Tenenbaum gradient (Tenengrad) [7][8]: This algorithm convolves an image with Sobel operators, and then sums the square of the gradient vector components.

$$
F_{\text {Tenengrad }}=\sum_{\text {Height Width }} \sum_{x}(x, y)^{2}+S_{y}(x, y)^{2}
$$

where $S_{x}(x, y)$ and $S_{y}(x, y)$ are the resultant images from convolution with the Sobel operators.

5) Sum of modified Laplace [9]: It sums the absolute values of the convolution of an image with Laplacian operators.

$$
F_{S M L}=\sum_{\text {Height Width }} \sum_{x}\left|L_{x}(x, y)\right|+\left|L_{y}(x, y)\right|
$$

6) Energy Laplace [10]: This algorithm convolves an image with the mask

$$
L=\left[\begin{array}{lll}
-1 & -4 & -1 \\
-4 & 20 & -4 \\
-1 & -4 & -1
\end{array}\right]
$$

to compute the second derivative $C(x, y)$. The final output is the sum of the squares of the convolution results.

$$
F_{\text {energy_Laplace }}=\sum_{\text {Height Width }} \sum_{\text {Wid }} C(x, y)^{2}
$$

7) Wavelet algorithm $W_{1}$ [11][12]: It uses the Daubechies D6 wavelet filter, applying both high-pass $(\mathrm{H})$ and low-pass (L) filtering to an image. The resultant image is divided into four sub-images LL, HL, LH, and HH. The algorithm sums the absolute values in the $\mathrm{HL}, \mathrm{LH}$, and $\mathrm{HH}$ regions. 


$$
F_{W 1}=\sum_{\text {Height Width }} \sum_{H L}\left|W_{H}(x, y)\right|+\left|W_{L H}(x, y)\right|+\left|W_{H H}(x, y)\right|
$$

8) Wavelet algorithm $W_{2}$ [11][12]: This algorithm sums the variances in the $\mathrm{HL}, \mathrm{LH}$, and $\mathrm{HH}$ regions. The mean values $\mu$ in each region are computed from absolute values.

$$
\begin{aligned}
& F_{W 2}=\frac{1}{H \cdot W} \sum_{\text {Height Width }} \sum_{H L}\left(\left|W_{H L}(x, y)\right|-\mu_{H L}\right)^{2} \\
& +\left(\left|W_{L H}(x, y)\right|-\mu_{L H}\right)^{2}+\left(\left|W_{H H}(x, y)\right|-\mu_{H H}\right)^{2}
\end{aligned}
$$

where $H$ and $W$ are image height and width.

9) Wavelet algorithm $W_{3}$ [11][12]: This algorithm differs from (F-8) in that the mean values $\bar{\mu}$ in the HL, LH, and $\mathrm{HH}$ regions are computed without using absolute values.

$$
\begin{gathered}
F_{W 3}=\frac{1}{H \cdot W} \sum_{\text {Height Width }} \sum_{H L}\left(W_{H}(x, y)-\bar{\mu}_{H L}\right)^{2}+ \\
\left(W_{L H}(x, y)-\bar{\mu}_{L H}\right)^{2}+\left(W_{H H}(x, y)-\bar{\mu}_{H H}\right)^{2}
\end{gathered}
$$

\section{B. Statistics-Based Algorithms}

These statistics-based algorithms, generally less sensitive to noise than derivative-based algorithms, distinguish focused images from defocused images using variance and correlation. 10) Variance [7][13]: This algorithm computes variations in grey level among image pixels. It uses the power function to amplify larger differences from the mean intensity $\mu$ instead of simply amplifying high intensity values.

$$
F_{\text {variance }}=\frac{1}{H \cdot W} \sum_{\text {Height Width }} \sum_{i}(i(x, y)-\mu)^{2}
$$

11) Normalized variance [7][13]: Normalizing the final output with the mean intensity $\mu$, this algorithm compensates for the differences in average image intensity among different images.

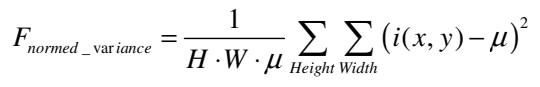

12) AutoCorrelation [4][5]:

$$
F_{\text {auto_corr }}=\sum_{\text {Height Width }} \sum_{i} i(x, y) \cdot i(x+1, y)-\sum_{\text {Height Width }} \sum_{i} i(x, y) \cdot i(x+2, y)
$$

13) Standard-deviation-based correlation [2][3]:

$$
F_{\text {corr_stddev }}=\sum_{\text {Height Width }} \sum_{i}(x, y) \cdot i(x+1, y)-H \cdot W \cdot \mu^{2}
$$

\section{Histogram-Based Algorithms}

These algorithms use histograms $h(i)$ (i.e., the number of pixels with intensity $i$ in an image) to analyze the distribution and frequency of image intensities.

14) Range algorithm [14]: This algorithm computes the difference between the highest and the lowest intensity levels.

$$
F_{\text {range }}=\max _{i}(h(i)>0)-\min _{i}(h(i)>0)
$$

15) Entropy algorithm [14]: It assumes that focused images contain more information than defocused images.

$$
F_{\text {entropy }}=-\sum_{\text {Intensities }} p_{i} \cdot \log _{2}\left(p_{i}\right)
$$

where $p_{i}=h(i) / H \cdot W$ is probability of a pixel with intensity $i$.

\section{Intuitive Algorithms}

16) Thresholded content [13][15]: This algorithm sums the pixel intensities above a threshold.

$$
F_{\text {th_cont }}=\sum_{\text {Height Width }} \sum_{i} i(x, y)
$$

where $i(x, y) \geq \theta$.

17) Thresholded pixel count [13]: This algorithm counts the number of pixels having intensity below a given threshold.

$$
F_{\text {pixel_count }}=\sum_{\text {Height Width }} \sum_{\text {W }} s(i(x, y), \theta)
$$

where $s(i(x, y), \theta)=\left\{\begin{array}{l}1, i(x, y) \leq \theta \\ 0, \text { else }\end{array}\right.$.

18) Image power [3]: This algorithm sums the square of image intensities above a given threshold.

$$
F_{\text {power }}=\sum_{\text {Height Width }} \sum_{i} i(x, y)^{2}
$$

where $i(x, y) \geq \theta$.

\section{IMAGE PRE-PROCESSING}

In order to test the behavior of the focus algorithms under different conditions, three approaches were individually used to pre-process images before a focus algorithm was applied.

1) Sub-sampling: Sub-sampling images increases execution speed because of reduced image data. Furthermore, it is also useful for testing a focus algorithm's robustness to image information reduction. In this study, a 50\% sub sampling means that every second pixel of an image is used both horizontally and vertically, resulting in 1/4 of the input data.

2) Adding random noise: In order to investigate the influence of noise on the performance of the focus algorithms, a random noise filter was applied to the images.

3) Low-pass filtering: A binomial filter was used to preprocess images to reduce image noise levels and to investigate the overall impact of low-pass filtering on the performance of the focus algorithms. The convolution vector was constructed from the binomial numbers of Pascal's Triangle. For example, the convolution vector of size five is $[1,4,6,4,1]$.

\section{RANKING METHODOLOGY}

Each focus algorithm produces a focus curve for an image set. In order to make valid comparisons, these focus curves were normalized and the curves inverted with their peak as the global minimum. The focus algorithms were evaluated using the following five criteria. The algorithms were ranked according to individual criteria as well as overall score.

1) Accuracy: This criterion measures the distance between the best focus position, manually determined by proficient microscope technicians, and the maximum of the focus curve. The smaller this measure is, the more accurate the algorithm.

2) Range: The range criterion describes the distance between two neighboring local minima around the global maximum. The larger this measure is, the easier it is to reach the global maximum without being trapped in a local maximum.

3) Number of false maxima: This criterion describes the number of maxima appearing in a focus curve, excluding the global maximum.

4) Width: This criterion describes the sharpness/narrowness of the peak. The width of a curve at $50 \%$ of its height is computed as the width of the focus curve. 
5) Noise level: Criteria similar to the above four criteria were adopted in [3] for evaluating focus algorithm performance for fluorescence microscopy applications. Besides these four criteria, the noise level of a curve was also found to be important for autofocusing in this study. This criterion extends the false maximum criterion by describing the speed of the direction changes (2nd derivative) between two false maxima of a focus curve. Two focus curves can have the same number of false maxima; however, the curve with local peaks of lower magnitude is better because the lower deviation from the ideal behavior increases the accuracy of approximation methods, such as forward Euler integration for adaptively setting step sizes and setting the next position of microscope's Z-motor.

In order to quantitatively represent this criterion, the squares of the second derivatives are summed. The derivatives are obtained by convolving the focus curve with the Laplacian operator $[-1,2,-1]$. The value at the peak is left out in order not to penalize algorithms with a small width.

6) Overall score: For each focus curve produced by a focus algorithm, the difference/distance of each individual criterion (i.e., accuracy, range, number of false maximum, width, and noise level) from the corresponding ideal values is computed. The ideal value for the Range criterion is the total number of images in an image set. In this study, there are 400 images in an image set under 100X and 550 images in an image set under $400 \mathrm{X}$. The ideal values for all the other criteria are 0 .

Distances for each criterion from their ideal values are defined for a focus algorithm $m$ as

$$
\begin{gathered}
\operatorname{Dist}_{\text {accuracy }}(m)=\operatorname{accuracy}(m) \\
\operatorname{Dist}_{\text {range }}(m)=\operatorname{maxRange}-\operatorname{range}(m) \\
\operatorname{Dist}_{\text {false_maxima }}(m)=\# \text { false maxima }(m) \\
\operatorname{Dist}_{\text {width }}(m)=\operatorname{width}(m) \\
\text { Dist }_{\text {noise_level }}(m)=\operatorname{noise} \text { level }(m)
\end{gathered}
$$

Consequently, an optimum focus curve is at $[0,0,0,0,0]$ in terms of criterion distances. In order to give all the distances equal weights, the criterion distances are all normalized. The lower a criterion distance, the better the performance of a focus algorithm under this criterion. The overall score is defined as the Euclidian distance of a focus curve to $[0,0,0,0,0]$. The lower this overall score, the better the overall performance of a focus algorithm.

$$
\operatorname{score}(m)=\sqrt{\sum_{\text {criteria }} \text { Dist }_{\text {criterion }}(m)^{2}}
$$

\section{RESUlTS AND DisCUSSIONS}

A total of 139,000 images were obtained on the six samples that were observed under three observation conditions (i.e., bright field, phase contrast, and DIC) and two magnifications (100X and 400X). A threshold of 150 was set for focus algorithms (F-15)-(F-18) because these algorithms exhibit satisfactory behavior with this threshold value. No threshold was set for the gradient-based algorithms (F-1)-(F-4) because threshold variation was not found to produce significant performance differences for these focus algorithms.
With criterion distances and the overall score defined in (1) and (2), lower values in all columns of the data tables in Appendix represent better performance. The italic numbers in parenthesis represent ranking of a focus algorithm according to individual criterion distances or the overall score.

\section{A. Without Image Pre-Processing}

1) Lumping observation methods and magnifications: Table 1 shows the performance and ranks of the 18 focus algorithms without image pre-processing according to individual criterion distances and the overall score. Each entry in the table is based on 36 image sets and averaged. One can see that Normalized Variance (F-11) and Standard-Deviation-Based Correlation (F13) have the best range, least number of false maxima, and the lowest noise level. By lumping the three observation methods and two magnifications and without applying image preprocessing, Normalized Variance (F-11) was found to provide the best overall performance.

2) Under different magnifications: Data shown in Table 2 and Table 3 were collected to investigate performance changes of the focus algorithms under different magnifications. No image pre-processing was conducted. The three observation methods were lumped together. Each entry in the tables is based on 18 image sets and averaged. By comparing the corresponding entries in Table 2 and Table 3, one can see that accuracy, range, number of false maxima, width, and noise level are almost consistently improved for all the focus algorithms under 100X magnification than under 400X magnification. In terms of the overall score, magnification differences do not significantly change the ranking of the focus algorithms. For example, Normalized Variance (F-11) provides the best overall performance under both $100 \mathrm{X}$ and $400 \mathrm{X}$.

As shown in Table 2 (also see Table 7), wavelet-based algorithms have small widths but always have local maxima around the global peak, which can be seen from the poor range distance. The small widths of the wavelet-based algorithms are due to the localized support property of wavelet basis, making them well suitable for image segmentation purposes [11].

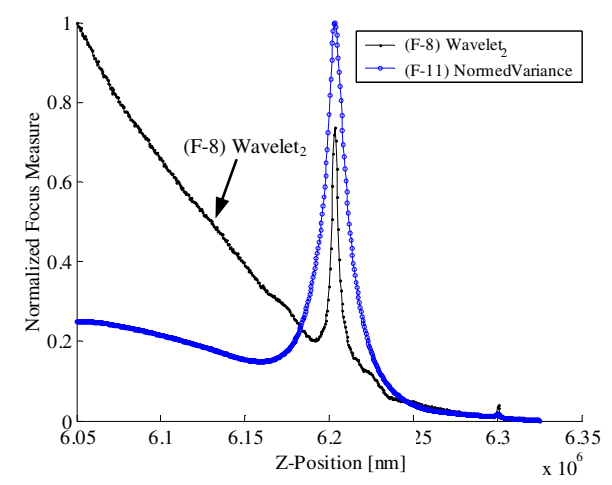

Fig. 4 Wavelet-based algorithm (F-8) with a misleading global maximum at the starting point. This undesired behavior only appears under 400X, which also disappears as sub sampling is applied to images (see Table 7).

By comparing Tables 2 and 3, one can see that the accuracy of Laplace (F-5 and F-6) and Wavelet-based algorithms (F-7, F-8, and F-9) significantly deteriorates under $400 X$. This occurs because these algorithms have a misleading 
global maximum at the starting point, as shown in Fig. 4. It should be noted that this undesired behavior only appears under 400X magnification, which also disappears as sub sampling is applied to images (see Table 7).

3) Using different observation methods: In order to investigate performance differences caused by observation methods, each criterion distance was computed based on 18 image sets for bright field, phase contrast, and DIC images. One can see from Table 4, 5, and 6 that most focus algorithms produce better accuracy under phase contrast than under bright field and DIC. It was also found in experiments that DIC images appear very similar to the gradient images under bright field, which improves the performance of the Image Power algorithm (F18). This can be seen by comparing the last rows of Table 4,5 , and 6. The improvement is due to the fact that Image Power (F-18) behaves similarly on DIC images as the squared gradient algorithm (F-2) on bright field images. By comparing the overall scores, Normalized Variance (F-11) was found to provide the best overall performance under all the three observation methods.

Interestingly, it was found that all the focus curves under phase contrast have two local peaks around the global peak (Fig. 5) due to the phase transition effect. The two local peaks limit the range of all the focus algorithms, without which the focus algorithms would be capable of producing much larger ranges (i.e., lower range distances).

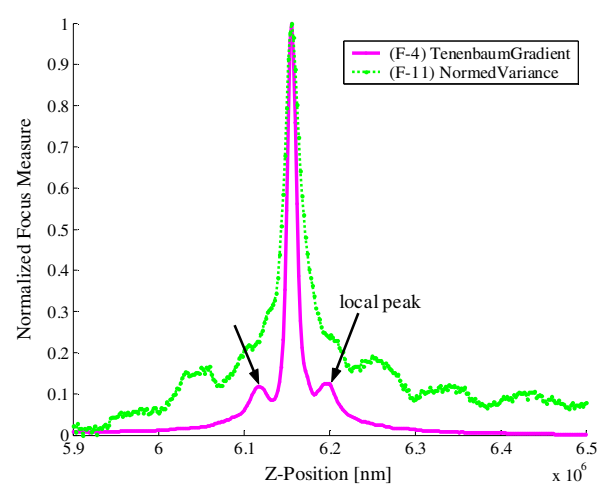

Fig. 5 Tennebaum Gradient (F-4) and Normalized Variance (F-11) on $5 \%$ sub sampled images under phase contrast. Phase transition effect is clearly visible with the two local peaks around the global peak.

\section{B. With Image Pre-Processing}

In order to investigate the influence of image preprocessing on the performance of the focus algorithms, the following pre-processing operations were individually applied.

(1) $100 \%$ (no sub sampling)

(2) $80 \%$ sub sampling of each image dimension

(3) $50 \%$ sub sampling of each image dimension

(4) $20 \%$ sub sampling of each image dimension

(5) $5 \%$ sub sampling of each image dimension

(6) 9 by 9 binomial filter on entire images

(7) noise filter with an intensity amplitude of 40 for adding random noise

It should be noted that in experiments the magnitudes of the binomial and noise filter were set to fairly large values in order to more visibly reveal the pre-processing effects.
Table 7 summarizes the effect of sub sampling on the criterion distances and overall scores. By comparing Table 1 (100\% sub sampled) and Table 7 (5\% sub sampled), one can see that (Normalized) Variance (F-10 and F-11) and Correlation-based algorithms (F-12 and F-13) are very sensitive to sub sampling. These algorithms (F-10)-(F-12) appear to behave as the Wavelet-based algorithms for $100 \%$ sub sampled images shown in Fig. 4, producing poor accuracy distances. The AutoCorrelation algorithm (F-12) does not exhibit this behavior, but it responds to sub sampling with a very high noise level. Generally, noise level was found to become worse for sub-sampled images because less information appears in the sub-sampled images. Widths remain relatively constant for the Wavelet-based algorithms (F-7)-(F9). These algorithms ((F-7)-(F-9)) rank the best in terms of widths, making them well suited for image segmentation purposes (Yang and Nelson, 2003a). One also finds from Table 8 that sub-sampling drastically reduces execution time.

Tenenbaum Gradient (F-4) was found to provide the best overall performance on sub-sampled (below 80\%) images because (F-4) uses more neighboring pixels than other algorithms such as Normalized Variance (F-11), making it more robust to reduced information content. As shown in Fig. 5 , (F-4) still performs satisfactorily on 5\% sub sampled images compared to $(\mathrm{F}-11)$. The two local peaks around the global peak are due to the phase transition effect.

Table 9 summarizes the effect of random noise on the criterion distances and overall score. Comparing Table 1 (no random noise added) and Table 9, one can see that the noise level increases dramatically when random noise is added. The criterion distances of false maxima and width also deteriorate. The accuracy of algorithms (F-4) (F-10) (F-11) and (F-13) is fairly constant, demonstrating their robustness to noise.

Table 10 summarizes the effect of low-pass filtering on the criterion distances and overall score. By comparing Table 1 (no low-pass pre-processing) and Table 10, one can see that the noise level decreases dramatically when low-pass preprocessing is applied to images. Accuracy and widths are improved for algorithms (F-5)-(F-9).

\section{CONCLUSION}

This paper presents a comprehensive comparison study of 18 focus algorithms. Six samples were used with three observation methods (bright field, phase contrast, and differential interference contrast (DIC)) under two magnifications (100X and 400X). A ranking methodology was proposed, based on which the 18 focus algorithms were ranked. Image pre-processing was also conducted to extensively reveal the performance and robustness of the focus algorithms. Based on the observations presented in the Results and Discussion section, one may conclude the following guidelines for selecting the optimal focus algorithm.

(1) For all three observation methods (i.e., bright field, phase contrast, and DIC), Normalized Variance (F-11) provides the best overall performance. Although AutoCorrelation (F-12) was found to be the optimal algorithm for 
fluorescence microscopy applications [3], it was not found to be capable of providing the best performance under bright field, phase contrast, or DIC.

(2) When images are sub sampled in order to increase execution speed, gradient-based focus algorithms should be selected, among which Tenenbaum Gradient (F-4) was found to provide the best overall performance.

(3) When images are noisy, Normalized Variance (F-11) is the most satisfactory due to its robustness to noise.

(4) If low pass filtering is applied as a pre-processing operation, Normalized Variance (F-11) is also capable of providing the best overall performance.

These general guidelines together with the observations presented in the Results and Discussion section allow for selecting the optimal focus algorithm for different microscopy applications. If more attention must be paid to a specific criterion instead of the overall performance under a particular set of conditions, for example, width for 5\% sub sampled images for image segmentation purposes, one can find that Wavelet-based focus algorithm (F-8) should be chosen by referring to Table 7 .

\section{REFERENCES}

[1] Y. Sun, B.J. Nelson, "Biological cell injection using an autonomous microrobotic system," International Journal of Robotics Research, Vol. 21, pp. 861-868, 2002.

[2] G. Yang, J.A. Gaines, B.J. Nelson, "A supervisory wafer-level 3D microassembly system for hybrid MEMS fabrications", Journal of Intelligent and Robotic Systems, Vol. 37, pp. 43-68, 2003.

[3] A. Santos, C.O. Solórzano, J.J. Vaquero, J.M. Peña, N. Malpica, F. Pozo, "Evaluation of autofocus functions in molecular cytogenetic analysis," Journal of Microscopy, Vol. 188, pp. 264-272, 1997.

[4] D. Vollath, "Automatic focusing by correlative methods," Journal of Microscopy, Vol. 147, pp. 279-288, 1987.

[5] D. Vollath, "The influence of the scene parameters and of noise on the behavior of automatic focusing algorithms," Journal of Microscopy, Vol. 151, pp. 133-146, 1988.

[6] J.F. Brenner, B.S. Dew, J.B. Horton, J.B. King, P.W. Neirath, W.D. Sellers, "An automated microscope for cytologic research," $J$. Histochem. Cytochem, Vol. 24, pp. 100-111, 1971.

[7] T. Yeo, S.O. Jayasooriah, R. Sinniah, "Autofocusing for tissue microscopy," Image Vision Comput., Vol. 11, pp. 629-639, 1993.

[8] E. Krotkov, "Focusing," Int. J. Comp. Vision, Vol. 1, pp. 223-237, 1987.

[9] S.K. Nayar, Y. Nakagawa, "Shape from focus," IEEE Trans. Pattern Anal. Machine Intell., Vol. 16, pp. 824-831, 1994.

[10] M. Subbarao, T.S. Choi, A. Nikzad, "Focusing techniques," J. Optical Engineering, Vol. 32, pp. 2824-2836, 1993.

[11] G. Yang, B.J. Nelson, "Wavelet-based auto-focusing and unsupervised segmentation of microscopic images," Proc. IEEE/RSJ International Conference on Intelligent Robots and Systems, 2003, pp. 2143-2148.

[12] G. Yang, B.J. Nelson, "Micromanipulation contact transition control by selective focusing and microforce control," Proc. IEEE International Conference on Robotics and Automation, 2003, pp. 3200-3206.

[13] F. Groen, I.T. Young, G. Ligthart, "A comparison of different focus functions for use in autofocus algorithms," Cytometry, Vol. 12, pp. 8191, 1985.

[14] L. Firestone, K. Cook, K. Culp, N. Talsania, K. Preston, "Comparison of autofocus methods for automated microscopy," Cytometry, Vol. 12, pp. 195-206, 1991.

[15] M.L. Mendelsohn, B.H. Mayall, "Computer-oriented analysis of human chromosomes," Comput. Biol. Med., Vol. 2, pp. 137-150, 1972.

\section{APPENDIX: DATA TABLES}

With criterion distances and the overall score defined in (1) and (2), lower values in all columns of the data tables in this section represent better performance. The italic numbers in parenthesis represent ranking of a focus algorithm according to individual criterion distances or the overall score.

TABLE 1 Ranking of the 18 focus algorithms according to individual criterion distances and overall score. Lumping the three observation methods and two magnifications, each entry is based on 36 image sets and averaged. No image pre-processing was conducted.

\begin{tabular}{|c|cc|cc|cc|cc|cc|cc|}
\hline Algorithm & \multicolumn{2}{|c|}{ Accuracy } & \multicolumn{2}{|c|}{ Range } & \multicolumn{2}{|c|}{ False Max } & \multicolumn{2}{|c|}{ Width } & \multicolumn{2}{|c|}{ Noise Level } & \multicolumn{2}{c|}{ Overall Score } \\
\hline F-1 & 2.83 & $(6)$ & 404.31 & $(8)$ & 110.56 & $(12)$ & 18.36 & $(5)$ & 0.0229 & $(4)$ & 1.1002 & $(7)$ \\
\hline F-2 & 3.03 & $(8)$ & 424.50 & $(11)$ & 110.33 & $(11)$ & 16.75 & $(4)$ & 0.0360 & $(8)$ & 1.1310 & $(8)$ \\
\hline F-3 & 2.69 & $(2)$ & 400.75 & $(6)$ & 98.17 & $(8)$ & 15.25 & $(3)$ & 0.0305 & $(6)$ & 1.0439 & $(5)$ \\
\hline F-4 & 2.69 & $(2)$ & 399.03 & $(4)$ & 95.61 & $(7)$ & 15.06 & $(2)$ & 0.0284 & $(5)$ & 1.0312 & $(4)$ \\
\hline F-5 & 15.67 & $(12)$ & 454.58 & $(13)$ & 139.50 & $(16)$ & 30.11 & $(6)$ & 0.1412 & $(14)$ & 1.3165 & $(14)$ \\
\hline F-6 & 14.64 & $(11)$ & 450.19 & $(12)$ & 127.75 & $(13)$ & 30.53 & $(8)$ & 0.0921 & $(12)$ & 1.2585 & $(11)$ \\
\hline F-7 & 16.03 & $(13)$ & 457.17 & $(15)$ & 142.06 & $(17)$ & 30.33 & $(7)$ & 0.1795 & $(16)$ & 1.3324 & $(16)$ \\
\hline F-8 & 29.47 & $(15)$ & 458.47 & $(16)$ & 129.03 & $(15)$ & 49.92 & $(10)$ & 0.1755 & $(15)$ & 1.3190 & $(15)$ \\
\hline F-9 & 22.83 & $(14)$ & 456.50 & $(14)$ & 128.92 & $(14)$ & 41.64 & $(9)$ & 0.1182 & $(13)$ & 1.2936 & $(12)$ \\
\hline F-10 & 2.78 & $(5)$ & 153.64 & $(3)$ & 14.33 & $(3)$ & 51.92 & $(12)$ & 0.0051 & $(3)$ & 0.4131 & $(3)$ \\
\hline F-11 & 2.72 & $(4)$ & 138.92 & $(1)$ & 12.89 & $(2)$ & 51.81 & $(11)$ & 0.0050 & $(2)$ & 0.3877 & $(1)$ \\
\hline F-12 & 2.67 & $(1)$ & 410.19 & $(10)$ & 106.47 & $(9)$ & 14.08 & $(1)$ & 0.0313 & $(7)$ & 1.0915 & $(6)$ \\
\hline F-13 & 2.83 & $(6)$ & 148.75 & $(2)$ & 12.58 & $(1)$ & 53.28 & $(13)$ & 0.0044 & $(1)$ & 0.4070 & $(2)$ \\
\hline F-14 & 6.44 & $(10)$ & 472.17 & $(17)$ & 109.72 & $(10)$ & 105.72 & $(17)$ & 0.5969 & $(17)$ & 1.3152 & $(13)$ \\
\hline F-15 & 5.22 & $(9)$ & 482.97 & $(18)$ & 156.17 & $(18)$ & 72.25 & $(14)$ & 3.6897 & $(18)$ & 1.7662 & $(18)$ \\
\hline F-16 & 56.22 & $(17)$ & 403.75 & $(7)$ & 51.78 & $(6)$ & 105.22 & $(16)$ & 0.0583 & $(10)$ & 1.1889 & $(10)$ \\
\hline F-17 & 94.06 & $(18)$ & 408.31 & $(9)$ & 45.78 & $(4)$ & 211.67 & $(18)$ & 0.0795 & $(11)$ & 1.6736 & $(17)$ \\
\hline F-18 & 55.22 & $(16)$ & 400.39 & $(5)$ & 51.25 & $(5)$ & 94.64 & $(15)$ & 0.0534 & $(9)$ & 1.1575 & $(9)$ \\
\hline
\end{tabular}

TABLE 2 Ranking of the 18 focus algorithms according to individual criterion distances under 100X magnification. Each entry is based on 18 image sets and averaged. No image pre-processing was conducted.

\begin{tabular}{|c|cc|cc|cc|cc|cc|cc|}
\hline Algorithm & \multicolumn{2}{|c|}{ Accuracy } & \multicolumn{2}{|c|}{ Range } & \multicolumn{2}{|c|}{ False Max } & \multicolumn{2}{|c|}{ Width } & \multicolumn{2}{c|}{ Noise Level } & \multicolumn{2}{c|}{ Overall Score } \\
\hline F-1 & 0.44 & $(4)$ & 339.83 & $(5)$ & 88.28 & $(10)$ & 10.44 & $(10)$ & 0.0214 & $(4)$ & 1.0444 & $(8)$ \\
\hline F-2 & 0.44 & $(4)$ & 363.22 & $(10)$ & 91.89 & $(12)$ & 6.83 & $(5)$ & 0.0444 & $(11)$ & 1.1036 & $(10)$ \\
\hline F-3 & 0.39 & $(2)$ & 349.33 & $(7)$ & 77.61 & $(8)$ & 7.50 & $(6)$ & 0.0366 & $(10)$ & 1.0137 & $(6)$ \\
\hline F-4 & 0.39 & $(2)$ & 350.00 & $(8)$ & 75.17 & $(7)$ & 7.72 & $(9)$ & 0.0330 & $(8)$ & 1.0048 & $(5)$ \\
\hline F-5 & 0.56 & $(9)$ & 382.89 & $(13)$ & 114.33 & $(16)$ & 6.67 & $(4)$ & 0.0555 & $(12)$ & 1.2483 & $(14)$ \\
\hline F-6 & 0.61 & $(13)$ & 382.78 & $(12)$ & 110.00 & $(13)$ & 5.39 & $(1)$ & 0.0767 & $(14)$ & 1.2262 & $(11)$ \\
\hline F-7 & 0.56 & $(9)$ & 385.44 & $(14)$ & 117.44 & $(17)$ & 6.50 & $(3)$ & 0.0620 & $(13)$ & 1.2687 & $(15)$ \\
\hline F-8 & 0.50 & $(7)$ & 387.39 & $(15)$ & 112.28 & $(15)$ & 7.50 & $(6)$ & 0.1022 & $(16)$ & 1.2462 & $(12)$ \\
\hline F-9 & 0.56 & $(9)$ & 388.06 & $(16)$ & 112.17 & $(14)$ & 5.83 & $(2)$ & 0.0894 & $(15)$ & 1.2464 & $(13)$ \\
\hline F-10 & 0.50 & $(7)$ & 155.11 & $(3)$ & 11.61 & $(3)$ & 45.50 & $(12)$ & 0.0072 & $(3)$ & 0.4636 & $(3)$ \\
\hline F-11 & 0.44 & $(4)$ & 131.94 & $(1)$ & 8.83 & $(1)$ & 45.44 & $(11)$ & 0.0071 & $(2)$ & 0.4171 & $(1)$ \\
\hline F-12 & 0.33 & $(1)$ & 358.39 & $(9)$ & 81.50 & $(9)$ & 7.67 & $(8)$ & 0.0344 & $(9)$ & 1.0480 & $(9)$ \\
\hline F-13 & 0.56 & $(9)$ & 150.94 & $(2)$ & 10.39 & $(2)$ & 47.56 & $(13)$ & 0.0061 & $(1)$ & 0.4614 & $(2)$ \\
\hline F-14 & 3.00 & $(14)$ & 408.67 & $(17)$ & 90.94 & $(11)$ & 107.56 & $(17)$ & 0.5626 & $(17)$ & 1.3514 & $(16)$ \\
\hline F-15 & 3.06 & $(15)$ & 420.33 & $(18)$ & 134.11 & $(18)$ & 54.50 & $(14)$ & 3.8237 & $(18)$ & 1.7617 & $(18)$ \\
\hline F-16 & 21.44 & $(17)$ & 339.94 & $(6)$ & 46.72 & $(5)$ & 81.83 & $(16)$ & 0.0226 & $(5)$ & 1.0335 & $(7)$ \\
\hline F-17 & 86.00 & $(18)$ & 368.94 & $(11)$ & 38.28 & $(4)$ & 170.44 & $(18)$ & 0.0306 & $(7)$ & 1.6888 & $(17)$ \\
\hline F-18 & 20.56 & $(16)$ & 332.28 & $(4)$ & 46.89 & $(6)$ & 67.83 & $(15)$ & 0.0293 & $(6)$ & 0.9812 & $(4)$ \\
\hline
\end{tabular}

TABLE 3 Ranking of the 18 focus algorithms according to individual criterion distances under $\mathbf{4 0 0 X}$ magnification. Each entry is based on 18 image sets and averaged. No image pre-processing was conducted.

\begin{tabular}{|c|cc|cc|cc|cc|cc|cc|}
\hline Algorithm & \multicolumn{2}{|c|}{ Accuracy } & \multicolumn{2}{|c|}{ Range } & \multicolumn{2}{|c|}{ False Max } & \multicolumn{2}{|c|}{ Width } & \multicolumn{2}{c|}{ Noise Level } & \multicolumn{2}{c|}{ Overall Score } \\
\hline F-1 & 5.22 & $(7)$ & 468.78 & $(10)$ & 132.83 & $(12)$ & 26.28 & $(4)$ & 0.0244 & $(5)$ & 1.1433 & $(7)$ \\
\hline F-2 & 5.61 & $(8)$ & 485.78 & $(11)$ & 128.78 & $(10)$ & 26.67 & $(5)$ & 0.0276 & $(7)$ & 1.1528 & $(8)$ \\
\hline F-3 & 5.00 & $(1)$ & 452.17 & $(6)$ & 118.72 & $(8)$ & 23.00 & $(3)$ & 0.0244 & $(6)$ & 1.0683 & $(5)$ \\
\hline F-4 & 5.00 & $(1)$ & 448.06 & $(5)$ & 116.06 & $(7)$ & 22.39 & $(2)$ & 0.0237 & $(4)$ & 1.0529 & $(4)$ \\
\hline F-5 & 30.78 & $(12)$ & 526.28 & $(14)$ & 164.67 & $(16)$ & 53.56 & $(6)$ & 0.2269 & $(14)$ & 1.3870 & $(14)$ \\
\hline F-6 & 28.67 & $(11)$ & 517.61 & $(12)$ & 145.50 & $(13)$ & 55.67 & $(8)$ & 0.1076 & $(11)$ & 1.3018 & $(10)$ \\
\hline F-7 & 31.50 & $(13)$ & 528.89 & $(15)$ & 166.67 & $(17)$ & 54.17 & $(7)$ & 0.2970 & $(16)$ & 1.4008 & $(15)$ \\
\hline F-8 & 58.44 & $(15)$ & 529.56 & $(16)$ & 145.78 & $(15)$ & 92.33 & $(14)$ & 0.2489 & $(15)$ & 1.4411 & $(16)$ \\
\hline F-9 & 45.11 & $(14)$ & 524.94 & $(13)$ & 145.67 & $(14)$ & 77.44 & $(12)$ & 0.1469 & $(13)$ & 1.3727 & $(12)$ \\
\hline F-10 & 5.06 & $(5)$ & 152.17 & $(3)$ & 17.06 & $(3)$ & 58.33 & $(10)$ & 0.0030 & $(3)$ & 0.3776 & $(3)$ \\
\hline F-11 & 5.00 & $(1)$ & 145.89 & $(1)$ & 16.94 & $(2)$ & 58.17 & $(9)$ & 0.0030 & $(2)$ & 0.3686 & $(1)$ \\
\hline F-12 & 5.00 & $(1)$ & 462.00 & $(7)$ & 131.44 & $(11)$ & 20.50 & $(1)$ & 0.0283 & $(8)$ & 1.1269 & $(6)$ \\
\hline F-13 & 5.11 & $(6)$ & 146.56 & $(2)$ & 14.78 & $(1)$ & 59.00 & $(11)$ & 0.0028 & $(1)$ & 0.3687 & $(2)$ \\
\hline F-14 & 9.89 & $(10)$ & 535.67 & $(17)$ & 128.50 & $(9)$ & 103.89 & $(15)$ & 0.6312 & $(17)$ & 1.3013 & $(9)$ \\
\hline F-15 & 7.39 & $(9)$ & 545.61 & $(18)$ & 178.22 & $(18)$ & 90.00 & $(13)$ & 3.5557 & $(18)$ & 1.7697 & $(18)$ \\
\hline F-16 & 91.00 & $(17)$ & 467.56 & $(8)$ & 56.83 & $(6)$ & 128.61 & $(17)$ & 0.0940 & $(10)$ & 1.3746 & $(13)$ \\
\hline F-17 & 102.11 & $(18)$ & 447.67 & $(4)$ & 53.28 & $(4)$ & 252.89 & $(18)$ & 0.1285 & $(12)$ & 1.6625 & $(17)$ \\
\hline F-18 & 89.89 & $(16)$ & 468.50 & $(9)$ & 55.61 & $(5)$ & 121.44 & $(16)$ & 0.0775 & $(9)$ & 1.3567 & $(11)$ \\
\hline
\end{tabular}


TABLE 4 Ranking of the 18 focus algorithms according to individual criterion distance and overall score. Each entry is based on 18 image sets under bright field and averaged. No image pre-processing was conducted.

\begin{tabular}{|c|cc|cc|cc|cc|cc|cc|}
\hline Algorithm & \multicolumn{2}{|c|}{ Accuracy } & \multicolumn{2}{|c|}{ Range } & \multicolumn{2}{|c|}{ False Max } & \multicolumn{2}{c|}{ Width } & \multicolumn{2}{c|}{ Noise Level } & \multicolumn{2}{c|}{ Overall Score } \\
\hline F-1 & 2.67 & $(9)$ & 417.00 & $(7)$ & 131.00 & $(12)$ & 16.17 & $(5)$ & 0.0225 & $(5)$ & 1.1979 & $(7)$ \\
\hline F-2 & 2.50 & $(5)$ & 428.50 & $(9)$ & 129.50 & $(11)$ & 15.33 & $(4)$ & 0.0325 & $(8)$ & 1.2083 & $(8)$ \\
\hline F-3 & 2.42 & $(3)$ & 408.92 & $(5)$ & 121.17 & $(9)$ & 14.08 & $(3)$ & 0.0246 & $(6)$ & 1.1425 & $(5)$ \\
\hline F-4 & 2.42 & $(3)$ & 406.00 & $(4)$ & 119.33 & $(8)$ & 14.00 & $(2)$ & 0.0224 & $(4)$ & 1.1303 & $(4)$ \\
\hline F-5 & 38.42 & $(14)$ & 462.08 & $(13)$ & 148.00 & $(16)$ & 37.67 & $(8)$ & 0.2763 & $(15)$ & 1.4048 & $(13)$ \\
\hline F-6 & 2.00 & $(1)$ & 459.67 & $(12)$ & 141.25 & $(13)$ & 32.58 & $(6)$ & 0.1438 & $(11)$ & 1.3158 & $(9)$ \\
\hline F-7 & 38.50 & $(15)$ & 465.67 & $(14)$ & 152.58 & $(17)$ & 37.58 & $(7)$ & 0.3649 & $(16)$ & 1.4301 & $(14)$ \\
\hline F-8 & 26.75 & $(12)$ & 469.50 & $(16)$ & 142.83 & $(14)$ & 54.75 & $(10)$ & 0.2533 & $(14)$ & 1.3851 & $(12)$ \\
\hline F-9 & 26.75 & $(12)$ & 467.08 & $(15)$ & 143.25 & $(15)$ & 50.25 & $(9)$ & 0.1798 & $(13)$ & 1.3776 & $(10)$ \\
\hline F-10 & 2.58 & $(6)$ & 135.92 & $(3)$ & 16.00 & $(3)$ & 67.50 & $(13)$ & 0.0029 & $(3)$ & 0.4813 & $(3)$ \\
\hline F-11 & 2.58 & $(6)$ & 125.50 & $(1)$ & 12.83 & $(1)$ & 66.92 & $(12)$ & 0.0027 & $(2)$ & 0.4626 & $(1)$ \\
\hline F-12 & 2.25 & $(2)$ & 416.33 & $(6)$ & 126.83 & $(10)$ & 13.42 & $(1)$ & 0.0278 & $(7)$ & 1.1777 & $(6)$ \\
\hline F-13 & 2.58 & $(6)$ & 126.83 & $(2)$ & 13.08 & $(2)$ & 69.08 & $(14)$ & 0.0026 & $(1)$ & 0.4742 & $(2)$ \\
\hline F-14 & 10.33 & $(11)$ & 477.50 & $(17)$ & 118.25 & $(7)$ & 101.42 & $(15)$ & 1.0535 & $(17)$ & 1.3787 & $(11)$ \\
\hline F-15 & 7.42 & $(10)$ & 484.92 & $(18)$ & 158.08 & $(18)$ & 63.75 & $(11)$ & 5.2433 & $(18)$ & 1.7698 & $(18)$ \\
\hline F-16 & 86.25 & $(17)$ & 456.33 & $(10)$ & 77.42 & $(5)$ & 128.83 & $(17)$ & 0.1205 & $(10)$ & 1.5359 & $(16)$ \\
\hline F-17 & 102.00 & $(18)$ & 422.25 & $(8)$ & 54.83 & $(4)$ & 179.00 & $(18)$ & 0.1585 & $(12)$ & 1.6969 & $(17)$ \\
\hline F-18 & 84.50 & $(16)$ & 458.67 & $(11)$ & 80.42 & $(6)$ & 121.75 & $(16)$ & 0.1183 & $(9)$ & 1.5175 & $(15)$ \\
\hline
\end{tabular}

TABLE 5 Ranking of the 18 focus algorithms according to individual criterion distance and overall score. Each entry is based on 18 image sets under phase contrast and averaged. No image pre-processing was conducted.

\begin{tabular}{|c|cc|cc|cc|cc|cc|cc|}
\hline Algorithm & \multicolumn{2}{|c|}{ Accuracy } & \multicolumn{2}{|c|}{ Range } & \multicolumn{2}{|c|}{ False Max } & \multicolumn{2}{|c|}{ Width } & \multicolumn{2}{|c|}{ Noise Level } & \multicolumn{2}{l|}{ Overall Score } \\
\hline F-1 & 2.58 & $(9)$ & 381.83 & $(4)$ & 73.17 & $(10)$ & 23.17 & $(8)$ & 0.0244 & $(4)$ & 0.9251 & $(6)$ \\
\hline F-2 & 3.33 & $(11)$ & 421.58 & $(10)$ & 85.58 & $(11)$ & 16.00 & $(2)$ & 0.0382 & $(9)$ & 1.0338 & $(8)$ \\
\hline F-3 & 2.50 & $(6)$ & 395.08 & $(5)$ & 64.67 & $(7)$ & 16.17 & $(3)$ & 0.0354 & $(7)$ & 0.9200 & $(5)$ \\
\hline F-4 & 2.50 & $(6)$ & 399.67 & $(6)$ & 60.83 & $(5)$ & 16.33 & $(4)$ & 0.0335 & $(6)$ & 0.9177 & $(4)$ \\
\hline F-5 & 4.17 & $(13)$ & 443.67 & $(12)$ & 124.83 & $(16)$ & 16.67 & $(5)$ & 0.0624 & $(11)$ & 1.2213 & $(14)$ \\
\hline F-6 & 2.50 & $(6)$ & 437.92 & $(11)$ & 114.08 & $(13)$ & 16.67 & $(5)$ & 0.0733 & $(13)$ & 1.1675 & $(10)$ \\
\hline F-7 & 4.83 & $(15)$ & 448.67 & $(15)$ & 128.33 & $(17)$ & 17.42 & $(7)$ & 0.0850 & $(14)$ & 1.2444 & $(15)$ \\
\hline F-8 & 2.08 & $(3)$ & 445.08 & $(13)$ & 117.50 & $(15)$ & 37.42 & $(10)$ & 0.2070 & $(16)$ & 1.2034 & $(13)$ \\
\hline F-9 & 2.33 & $(5)$ & 446.33 & $(14)$ & 115.83 & $(14)$ & 31.50 & $(9)$ & 0.1117 & $(15)$ & 1.1943 & $(12)$ \\
\hline F-10 & 1.92 & $(1)$ & 166.75 & $(3)$ & 7.92 & $(3)$ & 45.75 & $(12)$ & 0.0063 & $(3)$ & 0.4040 & $(3)$ \\
\hline F-11 & 1.92 & $(1)$ & 142.75 & $(1)$ & 6.42 & $(1)$ & 44.75 & $(11)$ & 0.0063 & $(2)$ & 0.3588 & $(1)$ \\
\hline F-12 & 2.58 & $(9)$ & 403.67 & $(7)$ & 69.92 & $(9)$ & 15.67 & $(1)$ & 0.0359 & $(8)$ & 0.9510 & $(7)$ \\
\hline F-13 & 2.08 & $(3)$ & 160.42 & $(2)$ & 7.58 & $(2)$ & 47.25 & $(13)$ & 0.0054 & $(1)$ & 0.3962 & $(2)$ \\
\hline F-14 & 4.42 & $(14)$ & 474.00 & $(16)$ & 105.33 & $(12)$ & 160.00 & $(17)$ & 0.4749 & $(17)$ & 1.3950 & $(16)$ \\
\hline F-15 & 3.92 & $(12)$ & 483.25 & $(18)$ & 155.92 & $(18)$ & 102.00 & $(14)$ & 3.2472 & $(18)$ & 1.7907 & $(18)$ \\
\hline F-16 & 41.67 & $(17)$ & 416.00 & $(9)$ & 61.83 & $(6)$ & 131.50 & $(16)$ & 0.0418 & $(10)$ & 1.1890 & $(11)$ \\
\hline F-17 & 99.67 & $(18)$ & 474.67 & $(17)$ & 65.25 & $(8)$ & 225.33 & $(18)$ & 0.0656 & $(12)$ & 1.7721 & $(17)$ \\
\hline F-18 & 40.67 & $(16)$ & 415.75 & $(8)$ & 57.17 & $(4)$ & 106.08 & $(15)$ & 0.0290 & $(5)$ & 1.1237 & $(9)$ \\
\hline
\end{tabular}

TABLE 6 Ranking of the 18 focus algorithms according to individual criterion distance and overall score. Each entry is based on 18 image sets under $\boldsymbol{D I C}$ and averaged. No image pre-processing was conducted.

\begin{tabular}{|c|cc|cc|cc|cc|cc|cc|}
\hline Algorithm & Accuracy & \multicolumn{2}{|c|}{ Range } & \multicolumn{2}{|c|}{ False Max } & \multicolumn{2}{c|}{ Width } & Noise Level & Overall Score \\
\hline F-1 & 3.25 & $(4)$ & 414.08 & $(10)$ & 127.50 & $(13)$ & 15.75 & $(4)$ & 0.0218 & $(7)$ & 1.1955 & $(10)$ \\
\hline F-2 & 3.25 & $(4)$ & 423.42 & $(11)$ & 115.92 & $(10)$ & 18.92 & $(5)$ & 0.0372 & $(11)$ & 1.1607 & $(8)$ \\
\hline F-3 & 3.17 & $(1)$ & 398.25 & $(8)$ & 108.67 & $(9)$ & 15.50 & $(3)$ & 0.0316 & $(10)$ & 1.0896 & $(7)$ \\
\hline F-4 & 3.17 & $(1)$ & 391.42 & $(7)$ & 106.67 & $(8)$ & 14.83 & $(2)$ & 0.0292 & $(8)$ & 1.0702 & $(6)$ \\
\hline F-5 & 4.42 & $(10)$ & 458.00 & $(15)$ & 145.67 & $(17)$ & 36.00 & $(6)$ & 0.0848 & $(15)$ & 1.3509 & $(13)$ \\
\hline F-6 & 39.42 & $(13)$ & 453.00 & $(12)$ & 127.92 & $(15)$ & 42.33 & $(8)$ & 0.0593 & $(12)$ & 1.3592 & $(14)$ \\
\hline F-7 & 4.75 & $(12)$ & 457.17 & $(14)$ & 145.25 & $(16)$ & 36.00 & $(6)$ & 0.0885 & $(16)$ & 1.3480 & $(12)$ \\
\hline F-8 & 59.58 & $(17)$ & 460.83 & $(16)$ & 126.75 & $(12)$ & 57.58 & $(17)$ & 0.0663 & $(14)$ & 1.4842 & $(16)$ \\
\hline F-9 & 39.42 & $(13)$ & 456.08 & $(13)$ & 127.67 & $(14)$ & 43.17 & $(10)$ & 0.0630 & $(13)$ & 1.3632 & $(15)$ \\
\hline F-10 & 3.83 & $(7)$ & 158.25 & $(2)$ & 19.08 & $(5)$ & 42.50 & $(9)$ & 0.0061 & $(3)$ & 0.3998 & $(3)$ \\
\hline F-11 & 3.67 & $(6)$ & 148.50 & $(1)$ & 19.42 & $(6)$ & 43.75 & $(12)$ & 0.0061 & $(2)$ & 0.3863 & $(1)$ \\
\hline F-12 & 3.17 & $(1)$ & 410.58 & $(9)$ & 122.67 & $(11)$ & 13.17 & $(1)$ & 0.0302 & $(9)$ & 1.1682 & $(9)$ \\
\hline F-13 & 3.83 & $(7)$ & 159.00 & $(3)$ & 17.08 & $(3)$ & 43.50 & $(11)$ & 0.0053 & $(1)$ & 0.3993 & $(2)$ \\
\hline F-14 & 4.55 & $(11)$ & 465.00 & $(17)$ & 105.58 & $(7)$ & 55.75 & $(15)$ & 0.2623 & $(17)$ & 1.2143 & $(11)$ \\
\hline F-15 & 4.33 & $(9)$ & 480.75 & $(18)$ & 154.50 & $(18)$ & 51.00 & $(13)$ & 2.5786 & $(18)$ & 1.7469 & $(18)$ \\
\hline F-16 & 40.75 & $(16)$ & 338.92 & $(6)$ & 16.08 & $(1)$ & 55.33 & $(14)$ & 0.0126 & $(4)$ & 0.9064 & $(5)$ \\
\hline F-17 & 80.50 & $(18)$ & 328.00 & $(5)$ & 17.25 & $(4)$ & 230.67 & $(18)$ & 0.0144 & $(6)$ & 1.5742 & $(17)$ \\
\hline F-18 & 40.50 & $(15)$ & 326.75 & $(4)$ & 16.17 & $(2)$ & 56.08 & $(16)$ & 0.0129 & $(5)$ & 0.8861 & $(4)$ \\
\hline
\end{tabular}

TABLE 7 Ranking of the 18 focus algorithms according to individual criterion distance and overall score. Each entry is based on 36 image sets and averaged. 5\% sub sampling was conducted.

\begin{tabular}{|c|cc|cc|cc|cc|cc|cc|}
\hline Algorithm & \multicolumn{2}{|c|}{ Accuracy } & \multicolumn{2}{|c|}{ Range } & \multicolumn{2}{|c|}{ False Max } & \multicolumn{2}{|c|}{ Width } & \multicolumn{2}{|c|}{ Noise Level } & Overall Score \\
\hline F-1 & 2.81 & $(2)$ & 464.69 & $(7)$ & 146.50 & $(11)$ & 18.92 & $(6)$ & 0.492 & $(6)$ & 1.3417 & $(3)$ \\
\hline F-2 & 24.03 & $(11)$ & 469.81 & $(9)$ & 147.61 & $(12)$ & 42.78 & $(9)$ & 1.176 & $(9)$ & 1.3880 & $(5)$ \\
\hline F-3 & 2.86 & $(3)$ & 463.33 & $(6)$ & 145.14 & $(10)$ & 18.69 & $(5)$ & 0.327 & $(3)$ & 1.3336 & $(2)$ \\
\hline F-4 & 2.69 & $(1)$ & 448.06 & $(4)$ & 126.22 & $(6)$ & 19.83 & $(7)$ & 0.044 & $(1)$ & 1.2306 & $(1)$ \\
\hline F-5 & 3.31 & $(4)$ & 473.64 & $(12)$ & 154.25 & $(14)$ & 22.11 & $(8)$ & 3.395 & $(14)$ & 1.3966 & $(7)$ \\
\hline F-6 & 60.89 & $(15)$ & 476.81 & $(15)$ & 149.97 & $(13)$ & 69.22 & $(10)$ & 1.466 & $(13)$ & 1.5364 & $(16)$ \\
\hline F-7 & 11.72 & $(9)$ & 476.67 & $(14)$ & 156.28 & $(16)$ & 10.31 & $(3)$ & 11.057 & $(17)$ & 1.4711 & $(12)$ \\
\hline
\end{tabular}

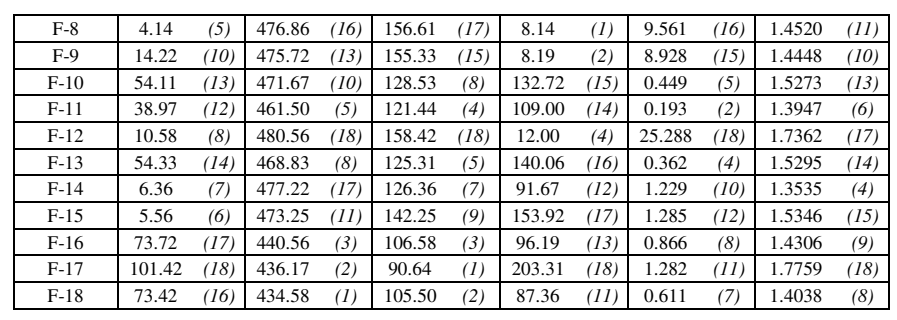

TABLE 8 Execution time decreases as more sub-sampling is applied.

\begin{tabular}{|c|c|c|c|c|}
\hline \multicolumn{2}{|c|}{ Algorithm } & $\mathbf{1 0 0} \%$ sampled & $\mathbf{8 0} \%$ sampled & 50\% sampled \\
\hline F-1 & ThresholdAbsGrad & $17 \mathrm{~ms}$ & $10 \mathrm{~ms}$ & $4 \mathrm{~ms}$ \\
\hline F-2 & SquaredGradient & $16 \mathrm{~ms}$ & $10 \mathrm{~ms}$ & $4 \mathrm{~ms}$ \\
\hline F-3 & BrennerGradient & $19 \mathrm{~ms}$ & $11 \mathrm{~ms}$ & $3 \mathrm{~ms}$ \\
\hline F-4 & TenenbaumGradient & $30 \mathrm{~ms}$ & $19 \mathrm{~ms}$ & $7 \mathrm{~ms}$ \\
\hline F-5 & ModifiedLaplace & $18 \mathrm{~ms}$ & $12 \mathrm{~ms}$ & $4 \mathrm{~ms}$ \\
\hline F-6 & EnergyLaplace & $33 \mathrm{~ms}$ & $21 \mathrm{~ms}$ & $8 \mathrm{~ms}$ \\
\hline F-7 & Wavelet_1 & $10 \mathrm{~ms}$ & $8 \mathrm{~ms}$ & $5 \mathrm{~ms}$ \\
\hline F-8 & Wavelet_2 & $16 \mathrm{~ms}$ & $12 \mathrm{~ms}$ & $6 \mathrm{~ms}$ \\
\hline F-9 & Wavelet_3 & $16 \mathrm{~ms}$ & $12 \mathrm{~ms}$ & $6 \mathrm{~ms}$ \\
\hline F-10 & Variance & $7 \mathrm{~ms}$ & $4 \mathrm{~ms}$ & $2 \mathrm{~ms}$ \\
\hline F-11 & NormedVariance & $7 \mathrm{~ms}$ & $4 \mathrm{~ms}$ & $1 \mathrm{~ms}$ \\
\hline F-12 & AutoCorrelation & $4 \mathrm{~ms}$ & $3 \mathrm{~ms}$ & $1 \mathrm{~ms}$ \\
\hline F-13 & StdDevCorr & $5 \mathrm{~ms}$ & $3 \mathrm{~ms}$ & $1 \mathrm{~ms}$ \\
\hline F-14 & Range & $3 \mathrm{~ms}$ & $2 \mathrm{~ms}$ & $<1 \mathrm{~ms}$ \\
\hline F-15 & Entropy & $6 \mathrm{~ms}$ & $4 \mathrm{~ms}$ & $1 \mathrm{~ms}$ \\
\hline F-16 & AboveThreshold & $4 \mathrm{~ms}$ & $2 \mathrm{~ms}$ & $1 \mathrm{~ms}$ \\
\hline F-17 & PixelCount & $3 \mathrm{~ms}$ & $2 \mathrm{~ms}$ & $1 \mathrm{~ms}$ \\
\hline F-18 & ImagePower & $5 \mathrm{~ms}$ & $3 \mathrm{~ms}$ & $1 \mathrm{~ms}$ \\
\hline
\end{tabular}

TABLE 9 Ranking of the 18 focus algorithms according to individual criterion distance and overall score. Each entry is based on 36 image sets and averaged. Random noise was added.

\begin{tabular}{|c|cc|cc|cc|cc|cc|cc|}
\hline Algorithm & \multicolumn{2}{|c|}{ Accuracy } & \multicolumn{2}{|c|}{ Range } & \multicolumn{2}{|c|}{ False Max } & \multicolumn{2}{|c|}{ Width } & \multicolumn{2}{|c|}{ Noise Level } & \multicolumn{2}{c|}{ Overall Score } \\
\hline F-1 & 14.36 & $(9)$ & 465.44 & $(9)$ & 149.50 & $(11)$ & 23.36 & $(5)$ & 0.7931 & $(10)$ & 1.3486 & $(8)$ \\
\hline F-2 & 14.31 & $(8)$ & 466.78 & $(10)$ & 149.78 & $(12)$ & 19.92 & $(4)$ & 1.1799 & $(11)$ & 1.3512 & $(9)$ \\
\hline F-3 & 2.28 & $(1)$ & 455.36 & $(6)$ & 142.03 & $(9)$ & 14.42 & $(2)$ & 0.1985 & $(6)$ & 1.2956 & $(6)$ \\
\hline F-4 & 2.67 & $(2)$ & 450.50 & $(5)$ & 141.08 & $(8)$ & 14.86 & $(3)$ & 0.1263 & $(4)$ & 1.2843 & $(5)$ \\
\hline F-5 & 50.19 & $(14)$ & 478.44 & $(14)$ & 156.00 & $(13)$ & 55.69 & $(13)$ & 8.6193 & $(15)$ & 1.4875 & $(14)$ \\
\hline F-6 & 35.03 & $(10)$ & 477.06 & $(13)$ & 156.08 & $(14)$ & 44.42 & $(8)$ & 7.8085 & $(14)$ & 1.4534 & $(12)$ \\
\hline F-7 & 44.11 & $(12)$ & 479.97 & $(16)$ & 159.44 & $(15)$ & 31.72 & $(6)$ & 24.468 & $(18)$ & 1.7537 & $(17)$ \\
\hline F-8 & 67.33 & $(16)$ & 479.97 & $(16)$ & 159.94 & $(17)$ & 39.78 & $(7)$ & 17.037 & $(17)$ & 1.6379 & $(16)$ \\
\hline F-9 & 63.50 & $(15)$ & 479.31 & $(15)$ & 159.44 & $(15)$ & 49.86 & $(9)$ & 9.2687 & $(16)$ & 1.5259 & $(15)$ \\
\hline F-10 & 2.75 & $(3)$ & 410.22 & $(1)$ & 99.69 & $(2)$ & 52.69 & $(10)$ & 0.0191 & $(3)$ & 1.0763 & $(2)$ \\
\hline F-11 & 2.75 & $(3)$ & 410.33 & $(2)$ & 99.17 & $(1)$ & 53.03 & $(11)$ & 0.0186 & $(2)$ & 1.0749 & $(1)$ \\
\hline F-12 & 2.78 & $(6)$ & 460.44 & $(7)$ & 145.64 & $(10)$ & 14.22 & $(1)$ & 0.5987 & $(9)$ & 1.3189 & $(7)$ \\
\hline F-13 & 2.75 & $(3)$ & 411.17 & $(3)$ & 99.92 & $(3)$ & 54.17 & $(12)$ & 0.0185 & $(1)$ & 1.0800 & $(3)$ \\
\hline F-14 & 35.94 & $(11)$ & 476.25 & $(12)$ & 113.67 & $(7)$ & 173.72 & $(17)$ & 2.4873 & $(12)$ & 1.4446 & $(11)$ \\
\hline F-15 & 11.06 & $(7)$ & 483.72 & $(18)$ & 159.97 & $(18)$ & 72.64 & $(14)$ & 7.6654 & $(13)$ & 1.4830 & $(13)$ \\
\hline F-16 & 86.17 & $(17)$ & 460.44 & $(7)$ & 106.53 & $(5)$ & 128.25 & $(16)$ & 0.2410 & $(8)$ & 1.3971 & $(10)$ \\
\hline F-17 & 156.64 & $(18)$ & 474.39 & $(11)$ & 110.69 & $(6)$ & 234.31 & $(18)$ & 0.2227 & $(7)$ & 1.8549 & $(18)$ \\
\hline F-18 & 47.69 & $(13)$ & 442.53 & $(4)$ & 101.50 & $(4)$ & 108.17 & $(15)$ & 0.1484 & $(5)$ & 1.2431 & $(4)$ \\
\hline
\end{tabular}

TABLE 10 Ranking of the 18 focus algorithms according to individual criterion distance and overall score. Each entry is based on 36 image sets and averaged. Low pass filtering was applied.

\begin{tabular}{|c|cc|cc|cc|cc|cc|cc|}
\hline Algorithm & \multicolumn{2}{|c|}{ Accuracy } & \multicolumn{2}{|c|}{ Range } & \multicolumn{2}{|c|}{ False Max } & \multicolumn{2}{c|}{ Width } & \multicolumn{2}{c|}{ Noise Level } & \multicolumn{2}{c|}{ Overall Score } \\
\hline F-1 & 2.89 & $(7)$ & 340.22 & $(4)$ & 70.17 & $(9)$ & 26.50 & $(10)$ & 0.0080 & $(4)$ & 0.8423 & $(4)$ \\
\hline F-2 & 2.83 & $(5)$ & 368.61 & $(7)$ & 75.08 & $(11)$ & 16.78 & $(4)$ & 0.0153 & $(8)$ & 0.9027 & $(7)$ \\
\hline F-3 & 2.83 & $(5)$ & 364.69 & $(6)$ & 72.92 & $(10)$ & 17.11 & $(7)$ & 0.0146 & $(6)$ & 0.8888 & $(6)$ \\
\hline F-4 & 2.78 & $(3)$ & 361.06 & $(5)$ & 69.69 & $(7)$ & 17.08 & $(6)$ & 0.0142 & $(5)$ & 0.8719 & $(5)$ \\
\hline F-5 & 2.78 & $(3)$ & 435.00 & $(13)$ & 109.94 & $(14)$ & 24.00 & $(8)$ & 0.0299 & $(9)$ & 1.1440 & $(12)$ \\
\hline F-6 & 2.47 & $(1)$ & 426.56 & $(12)$ & 108.75 & $(13)$ & 16.61 & $(3)$ & 0.0343 & $(10)$ & 1.1226 & $(9)$ \\
\hline F-7 & 3.00 & $(9)$ & 449.19 & $(14)$ & 122.69 & $(15)$ & 24.44 & $(9)$ & 0.0517 & $(12)$ & 1.2174 & $(14)$ \\
\hline F-8 & 3.33 & $(12)$ & 456.58 & $(17)$ & 136.31 & $(17)$ & 16.36 & $(2)$ & 0.1080 & $(17)$ & 1.2829 & $(16)$ \\
\hline F-9 & 2.94 & $(8)$ & 453.33 & $(16)$ & 130.08 & $(16)$ & 17.03 & $(5)$ & 0.0619 & $(14)$ & 1.2515 & $(15)$ \\
\hline F-10 & 3.28 & $(11)$ & 159.14 & $(2)$ & 12.78 & $(2)$ & 61.14 & $(12)$ & 0.0023 & $(3)$ & 0.4464 & $(2)$ \\
\hline F-11 & 3.14 & $(10)$ & 139.92 & $(1)$ & 11.14 & $(1)$ & 60.94 & $(11)$ & 0.0021 & $(1)$ & 0.4153 & $(1)$ \\
\hline F-12 & 2.72 & $(2)$ & 373.47 & $(8)$ & 80.25 & $(12)$ & 16.33 & $(1)$ & 0.0153 & $(7)$ & 0.9285 & $(8)$ \\
\hline F-13 & 3.42 & $(13)$ & 159.14 & $(2)$ & 12.81 & $(3)$ & 61.92 & $(13)$ & 0.0022 & $(2)$ & 0.4489 & $(3)$ \\
\hline F-14 & 6.53 & $(15)$ & 451.97 & $(15)$ & 70.14 & $(8)$ & 97.47 & $(15)$ & 0.1080 & $(16)$ & 1.1350 & $(11)$ \\
\hline F-15 & 5.47 & $(14)$ & 483.39 & $(18)$ & 157.83 & $(18)$ & 70.61 & $(14)$ & 3.9902 & $(18)$ & 1.7646 & $(18)$ \\
\hline F-16 & 52.19 & $(16)$ & 385.06 & $(10)$ & 51.19 & $(5)$ & 118.33 & $(17)$ & 0.0515 & $(11)$ & 1.1488 & $(13)$ \\
\hline F-17 & 100.81 & $(18)$ & 407.67 & $(11)$ & 51.94 & $(6)$ & 211.92 & $(18)$ & 0.0833 & $(15)$ & 1.6793 & $(17)$ \\
\hline F-18 & 55.25 & $(17)$ & 378.58 & $(9)$ & 50.31 & $(4)$ & 105.08 & $(16)$ & 0.0541 & $(13)$ & 1.1231 & $(10)$ \\
\hline & & & & & & & & & & & & \\
\hline
\end{tabular}

